\title{
Preparation and properties of poly(vinylidene fluoride) nanocomposites blended with graphene oxide coated silica hybrids
}

\author{
J. C. Wang, P. Chen, L. Chen, K. Wang, H. Deng, F. Chen, Q. Zhang*', Q. Fu \\ College of Polymer Science and Engineering, State Key Laboratory of Polymer Materials Engineering, Sichuan \\ University, Chengdu 610065, People's Republic of China
}

Received 3 August 2011; accepted in revised form 27 October 2011

\begin{abstract}
Graphene oxide coated silica hybirds ( $\left.\mathrm{SiO}_{2}-\mathrm{GO}\right)$ were fabricated through electrostatic assembly in this work, then blended with poly(vinylidene fluoride) (PVDF) by solution mixing to make PVDF nanocomposites. The interfacial interaction was investigated by scanning electron microscopy (SEM), polarized optical microscopy (POM) and Fourier transform infrared spectroscopy (FTIR). The results showed that the interfacial interaction was enhanced by adding of $\mathrm{SiO}_{2}-\mathrm{GO}$ and strong hydrogen bonds were observed. The as-made nanocomposites were investigated using standard tensile test and dynamic mechanical analysis (DMA) measurements, mechanical properties of PVDF with $\mathrm{SiO}_{2}$-GO hybrids showed limited improvement.
\end{abstract}

Keywords: polymer composites, graphene oxide, silica, interfacial interaction, hydrogen bonds

\section{Introduction}

Graphene is a monolayer graphite, discovered in 2004 [1]. The two-dimensional (2D) sheet carbon material has attracted much attention due to its unique properties, such as high mechanical properties [2], electronic transfer [3], thermal conductivity [4] and specific surface area [5]. Various methods have been explored to make graphene sheets, like chemical vapor deposition, epitaxial growth, mechanical or chemical exfoliations. Graphene oxide (GO) is derived from the oxidation of natural graphite flakes followed by ultrasonic treatment of the suspension. After oxidation, basal planes of GO sheets are decorated mostly with epoxide, hydroxyl, carbonyl, and carboxyl groups [6, 7]. GO is an active material, it can act as surfactant [8], covalently combined with other materials [9], etc. In recent years, graphene hybrid nanoparticles appeared [10-12], the active GO sheets can be used to modify conventional nanoparticles which were used in polymer nanocomposites.

PVDF is a polar polymer with excellent chemical, mechanical and electrical properties [13-15]. PVDF has been widely used in many fields, such as ultrafiltration and microfiltration membranes, electrode binder in lithium ion batteries, microwave transducers and its unique applications as piezoelectric and pyroelectric materials. It is well known that nanoparticles are usually used to enhance the properties of polymer by simple blending [16-19], the interfacial interaction between polymer and nanoparticles is crucial to the enhancement. However, Jae-Wan Kim et al. studied the morphology, crystalline structure and mechanical properties of PVDF composites blended with silica, the composites performance poor properties. The author considered that there was

\footnotetext{
${ }^{*}$ Corresponding author, e-mail: qinzhang@scu.edu.cn
}

(c) BME-PT 
no interaction between silica and PVDF [20]. Thus, the modification of silica in order to achieve a better interfacial interaction is necessary.

In this work, graphene oxide coated silica nanoparticles $\left(\mathrm{SiO}_{2}-\mathrm{GO}\right)$ were fabricated by electrostatic assembly, these nanoparticles were solution blended with PVDF, the interfacial interaction and mechanical properties were investigated.

\section{Experiments}

\subsection{Materials}

Graphite powders were purchased from Qingdao Black Dragon graphite Co., Ltd, China. Sub-micro sized silica (average size $=500 \mathrm{~nm}$ ) was purchased from Admatechs Co., Ltd, Japan. Potassium permanganate $\left(\mathrm{KMnO}_{4}\right)$, sulfuric acid $\left(\mathrm{H}_{2} \mathrm{SO}_{4}, 98 \%\right)$, sodium nitrate $\left(\mathrm{NaNO}_{3}\right)$ and hydrogen peroxide $\left(\mathrm{H}_{2} \mathrm{O}_{2}\right)$ were purchased from Kermel Chemical reagent plant (Chengdu, China), all reagents were used as received. 3-aminopropyltriethoxysilane (APS) was provided from commercial sources and used without purification. PVDF (FR901) was purchased from Shanghai 3F New Material Co., Ltd. China.

\subsection{Preparation of graphene oxide coated silica hybrids}

The surface modification of $\mathrm{SiO}_{2}$ with APS coupling agent was carried out in liquid phase. In a typical process, $\mathrm{SiO}_{2}$ powder $(10 \mathrm{~g})$ was firstly added to $150 \mathrm{~mL}$ ethanol, premixed and then ground by planetary ball mill with zirconia balls $(400 \mathrm{rpm}$, $30 \mathrm{~min}$ ) to get well dispersed $\mathrm{SiO}_{2}$ suspension. After the addition of another $150 \mathrm{~mL}$ ethanol, $0.5 \mathrm{~mL}$ silane coupling agent APS was added into the suspension. The mixture was stirred, heated up to $50^{\circ} \mathrm{C}$ for $12 \mathrm{~h}$, the obtained particles were filtered from the mixture, washed with ethanol and deionized water five times and dried under vacuum.

GO was made of natural graphite by Hummer's method [21]. The size of GO monolayer sheets were mostly $2 \sim 6 \mu \mathrm{m}$, the thickness was about $1.3 \mathrm{~nm}$. Exfoliation of GO was achieved by sonication for $2 \mathrm{~h}$ in an aqueous solvent, followed by centrifugation to remove the unexfoliated GO sheets $(3000 \mathrm{rpm}$, $30 \mathrm{~min}$ ). A diluted and well dispersed GO colloid solution with a concentration of $0.4 \mathrm{mg} / \mathrm{mL}$ was employed in the succeeding process.

$\mathrm{GO}$ coated silica hybrids $\left(\mathrm{SiO}_{2}-\mathrm{GO}\right)$ were fabricated by simple mixing positively charged $\mathrm{SiO}_{2}-\mathrm{NH}_{2}$ particles and negatively charged GO dispersion. As in a typical process, $400 \mathrm{~mL} \mathrm{SiO} 2-\mathrm{NH}_{2}(20 \mathrm{mg} / \mathrm{mL})$ were added into a $200 \mathrm{~mL}$ aqueous $\mathrm{GO}$ suspension $(0.4 \mathrm{mg} / \mathrm{mL})$ under mild magnetic stirring for $1 \mathrm{~h}$. Stopped stirring when the aqueous solution became transparent, then GO precipitated with $\mathrm{SiO}_{2}-\mathrm{NH}_{2}$ at the bottom of the beaker. The sediment solid $\left(\mathrm{SiO}_{2}-\right.$ GO hybrids) were collected and washed with water for several times to remove the unbound GO and afterwards dried under $60^{\circ} \mathrm{C}$ and grounded before use.

\subsection{Fabrication of $\mathrm{PVDF} / \mathrm{SiO}_{2}-\mathrm{GO}$ composites}

Different amounts of $\mathrm{SiO}_{2}-\mathrm{GO}$ and $\mathrm{SiO}_{2}$ were firstly added to $50 \mathrm{~mL}$ dimethyl acetylamide (DMAc) respectively, the solution was sonicated for $4 \mathrm{~h}$ and then transferred to a flask for mechanical stirring. After that, $8 \mathrm{~g}$ PVDF were added to the solution in batches ( 4 batches, 2 g every $30 \mathrm{~min}$ ), stirred at $60^{\circ} \mathrm{C}$ for $12 \mathrm{~h}$ to get homogenous solution. Subsequently, the solution was co-precipitated with deionized water and dried in an oven at $60^{\circ} \mathrm{C}$ for $24 \mathrm{~h}$ to remove remaining solvent, the precipitate was heat compressed at $210^{\circ} \mathrm{C}$. Pure PVDF was fabricated in the same way for comparison. The weight ratios of the nanoparticles in the composites were 5, 10, 15, $20 \%$ (Filler/PVDF) respectively.

\subsection{Characterization}

Morphological studies were carried out using a scanning electron microscope (SEM, JEOL JSM$5900 \mathrm{LV}$ ) in order to investigate the interface between nanofillers and PVDF matrix in the composites. The SEM images were obtained on the fracture surface of samples at an accelerating voltage of $20 \mathrm{kV}$. Transmission electron microscopy (TEM) observations were carried out to examine the micro-morphology of hybrids on a Tecnai F20 S-TWIN TEM under an acceleration voltage of $200 \mathrm{kV}$.

Morphological observations on crystallites of PVDF composites were conducted on a polarized optical microscopy (POM, Leica DMLP) equipped with a hot stage (Linkam THMS 600) under crossed polarizers. All samples were first inserted between two microscope cover slips and squeezed at $210^{\circ} \mathrm{C}$ to obtain a slice with a thickness around $30 \mu \mathrm{m}$. Subsequently, the as-prepared slice was transferred to the hot stage and held at $210^{\circ} \mathrm{C}$ for 3 minutes to 
achieve thermal equilibrium. It was followed by cooling to $40^{\circ} \mathrm{C}$ at $2^{\circ} \mathrm{C} / \mathrm{min}$. The POM micrographs were recorded by a digital camera.

Infrared spectra from 4000 to $650 \mathrm{~cm}^{-1}$ were obtained by a Nicolet 6700 Fourier Transform Infrared Spectroscopy (FTIR) instrument using ATR method, 32 accumulated cycles, 4 accuracy.

The dynamic properties of the materials were investigated using a Dynamic Mechanical Analyzer (DMA, TA Q800). The mode was Film-Tension, preload force was $0.01 \mathrm{~N}$, amplitude was $10 \mu \mathrm{m}$, frequency was $1 \mathrm{~Hz}$ and heating rate was $3^{\circ} \mathrm{C} / \mathrm{min}$. Standard tensile tests were performed using a tensile testing machine (SANSI, ShenZhen, China) with a cross-head speed of $5 \mathrm{~mm} / \mathrm{min}$ at room temperature $\left(23^{\circ} \mathrm{C}\right)$. The tensile strength at yield was determined according to GB/T 1040-92 standard. The values of the tensile strength were calculated as averages over 5 specimens for each composition.

\section{Results and discussion}

\subsection{Self-assembly of graphene oxide coated silica}

The overall synthetic procedure of graphene oxide coated silica was demonstrated in Figure 1, graphene oxide was negatively charged because of its plenty of functional groups such as carboxylic acid and phenolic hydroxy groups. Raw $\mathrm{SiO}_{2}$ particles were modified with APS, then it could form positively charged $\mathrm{SiO}_{2}-\mathrm{NH}_{3}{ }^{+}$particles due to the ionization of amino groups [22]. Therefore, $\mathrm{GO}$ sheets were easy to self assemble with $\mathrm{SiO}_{2}-\mathrm{NH}_{3}{ }^{+}$by electrostatic force and the $\mathrm{SiO}_{2}-\mathrm{GO}$ hybrids were obtained.
Raw $\mathrm{SiO}_{2}$ and $\mathrm{SiO}_{2}-\mathrm{GO}$ powders were shown in Figure $2 \mathrm{a}$ and $2 \mathrm{~b}$ respectively, it is well known that the colour of graphene oxide is brown, $\mathrm{SiO}_{2}-\mathrm{GO}$ was brown compared with white raw $\mathrm{SiO}_{2}$, indicating the existence of graphene oxide. However, was silica coated with graphene oxide? SEM photographs of raw $\mathrm{SiO}_{2}$ and $\mathrm{SiO}_{2}-\mathrm{GO}$ at the same magnification were shown in Figure $2 \mathrm{~d}$ and $2 \mathrm{e}$ respectively. Compared with the smooth surface of $\mathrm{SiO}_{2}$, the surface of $\mathrm{SiO}_{2}-\mathrm{GO}$ was rough and somewhat wrinkled. It was more obvious at the TEM image of $\mathrm{SiO}_{2}$-GO (Figure 2c), ultrathin graphene oxide sheets coated the $\mathrm{SiO}_{2}$ particles. Therefore, the experimental results above all proved that the $\mathrm{SiO}_{2}-\mathrm{GO}$ hybrids have been successfully made.

\subsection{Interfacial interaction between PVDF and nanofillers}

In order to study the interfacial interaction between PVDF and nanofillers, SEM photographs were observed. As shown in Figure 3a and $3 b$ were PVDF with $\mathrm{SiO}_{2}$ at low and high magnification respectively, a large number of $\mathrm{SiO}_{2}$ was pulled out of the PVDF matrix and left many holes on the fracture surface, the few number of $\mathrm{SiO}_{2}$ that was left in the fracture surface showed very smooth surface, and the gaps between PVDF matrix and $\mathrm{SiO}_{2}$ were obvious clear, these all indicated that the interfacial interaction between PVDF matrix and $\mathrm{SiO}_{2}$ was very poor. However, compared with $\mathrm{PVDF} / \mathrm{SiO}_{2}$, there were fewer holes in the fracture surface of $\mathrm{PVDF} /$ $\mathrm{SiO}_{2}$-GO (Figure $3 \mathrm{c}$ and 3d), PVDF was somewhat adhered to the $\mathrm{SiO}_{2}-\mathrm{GO}$ particles, the combination

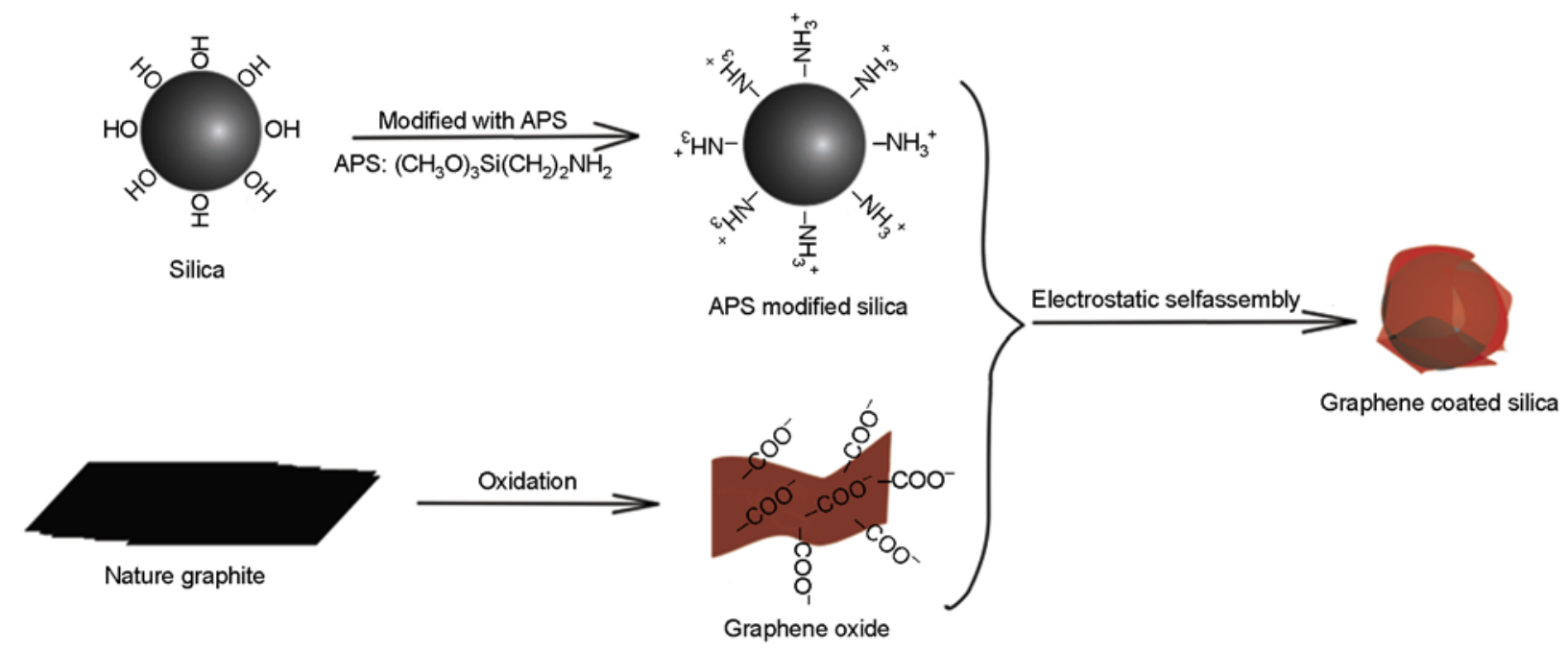

Figure 1. Illustration of the overall synthetic procedure of graphene oxide coated silica 


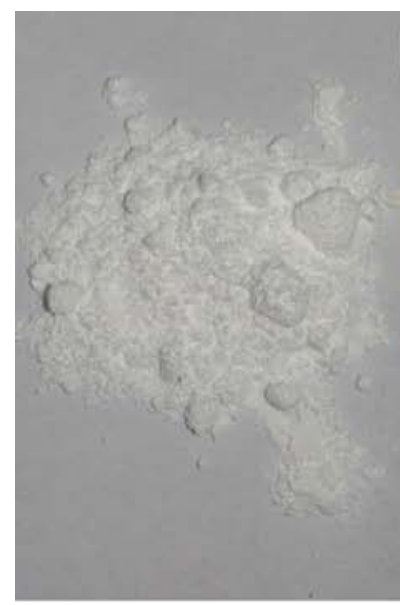

a)

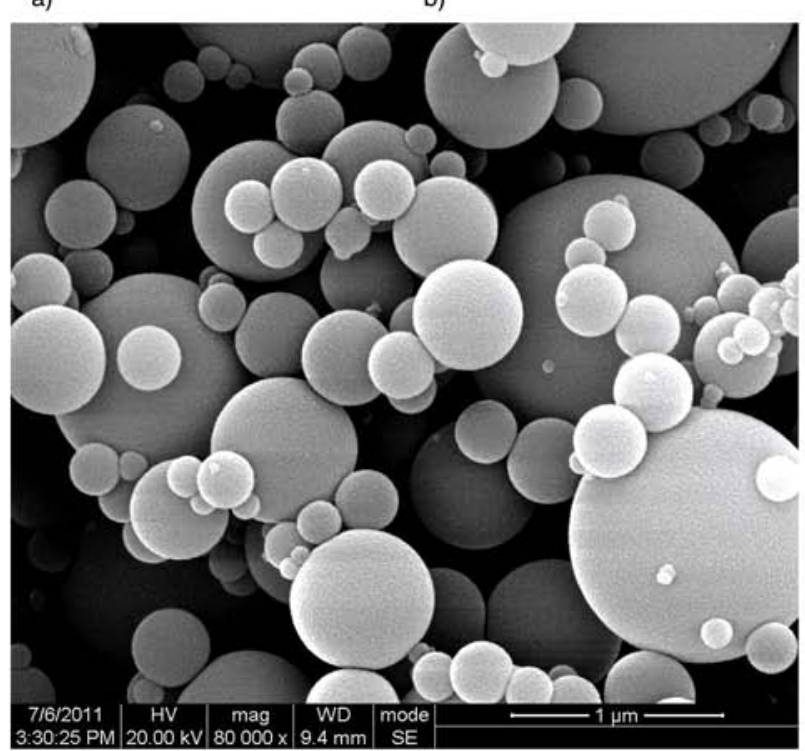

d)

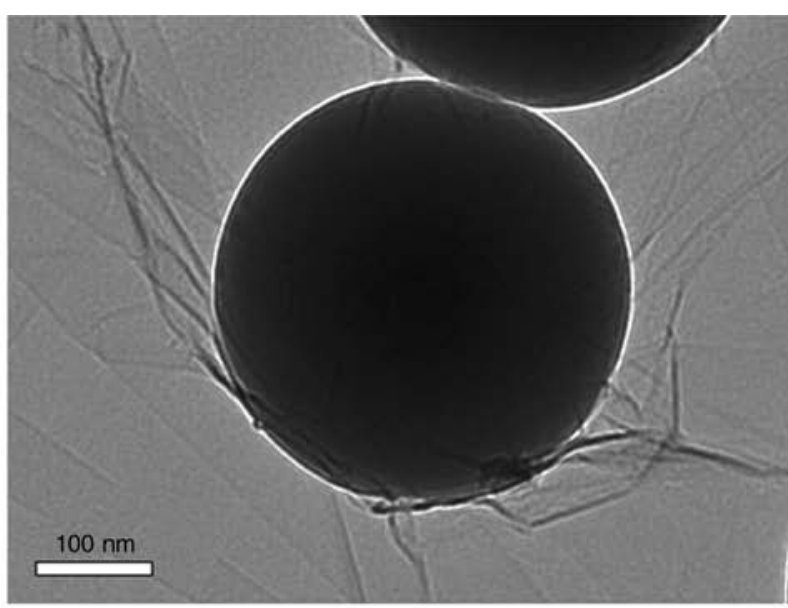

c)

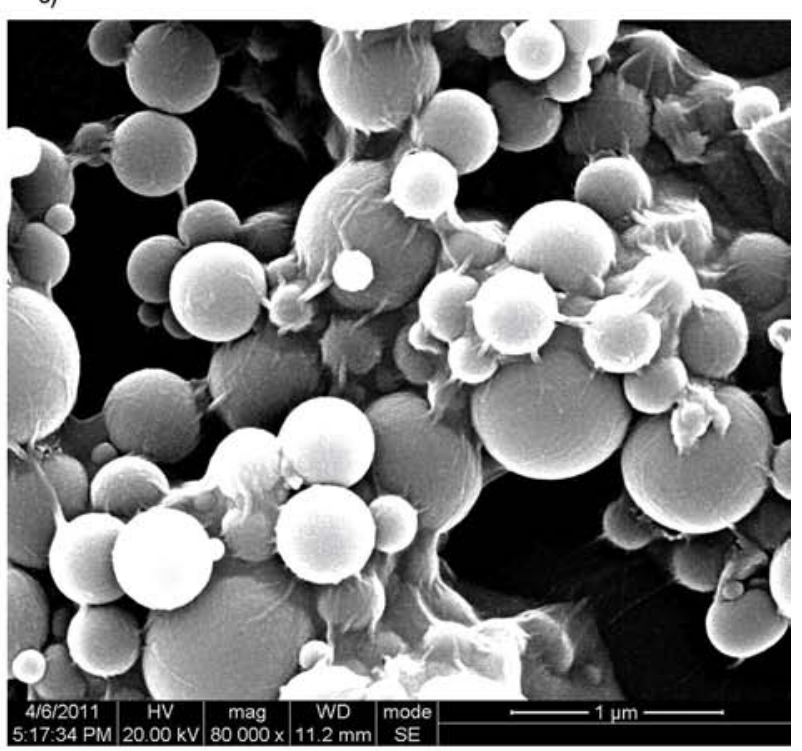

e)

Figure 2. Digital photographs of raw $\mathrm{SiO}_{2}$ (a) and as-made $\mathrm{SiO}_{2}-\mathrm{GO}$ hybrid (b), Transmission electron microscopy (TEM) image of $\mathrm{SiO}_{2}-\mathrm{GO}$ hybrids (c), scanning electron microscope (SEM) images of the raw $\mathrm{SiO}_{2}(\mathrm{~d})$ and created $\mathrm{SiO}_{2}$ $\mathrm{GO}$ hybrid (e) at the same magnification

between matrix and $\mathrm{SiO}_{2}-\mathrm{GO}$ was better. Therefore, after the silica was coated with graphene oxide, the interfacial interaction was enhanced in PVDF/ $\mathrm{SiO}_{2}-\mathrm{GO}$ composites.

The enhancement of interfacial interaction in $\mathrm{PVDF} / \mathrm{SiO}_{2}-\mathrm{GO}$ was also proved by another characterization method. It was reasonable that the plenty of functional groups like epoxide, hydroxyl, carbonyl, and carboxyl groups would have a strong interaction with the PVDF's molecular chains. The $\mathrm{C}-\mathrm{F}$ bond of PVDF and hydroxyl or carboxyl groups of graphene oxides were supposed to form hydrogen bonds in the $\mathrm{PVDF} / \mathrm{SiO}_{2}-\mathrm{GO}$ composites, the hydrogen bonds would support the enhancement of interfacial interaction. It is well known that the characteristic peaks in infrared spectra will show 'red shift' when hydrogen bonds are formed. Therefore, FTIR investigation (Figure 4) was made to prove this idea, $\mathrm{SiO}_{2}$ showed a strong peak at $1112.6 \mathrm{~cm}^{-1}$, which could be assigned as $\mathrm{Si}-\mathrm{O}-\mathrm{Si}$ vibration, while $\mathrm{PVDF} / 15 \% \mathrm{SiO} 2$ showed a peak of $\mathrm{Si}-\mathrm{O}-\mathrm{Si}$ vibration at $1107.3 \mathrm{~cm}^{-1}$, which implied slight red shift, because a few hydrogen bonds were formed. The peak of $\mathrm{Si}-\mathrm{O}-\mathrm{Si}$ vibration of $\mathrm{SiO}_{2}-\mathrm{GO}$ was located at $1117.3 \mathrm{~cm}^{-1}$, while that of PVDF/ $15 \% \mathrm{SiO}_{2}-\mathrm{GO}$ was located at $1104.7 \mathrm{~cm}^{-1}$. The obvious red shift indicated that there were substantial hydrogen bonds in the $\mathrm{PVDF} / \mathrm{SiO}_{2}-\mathrm{GO}$ composites, which could be attributed to the plenty of functional groups of the graphene oxide. Thus, compared to the $\mathrm{PVDF} / \mathrm{SiO}_{2}$ composites, the substantial hydrogen bonds between PVDF and $\mathrm{SiO}_{2}-\mathrm{GO}$ would 


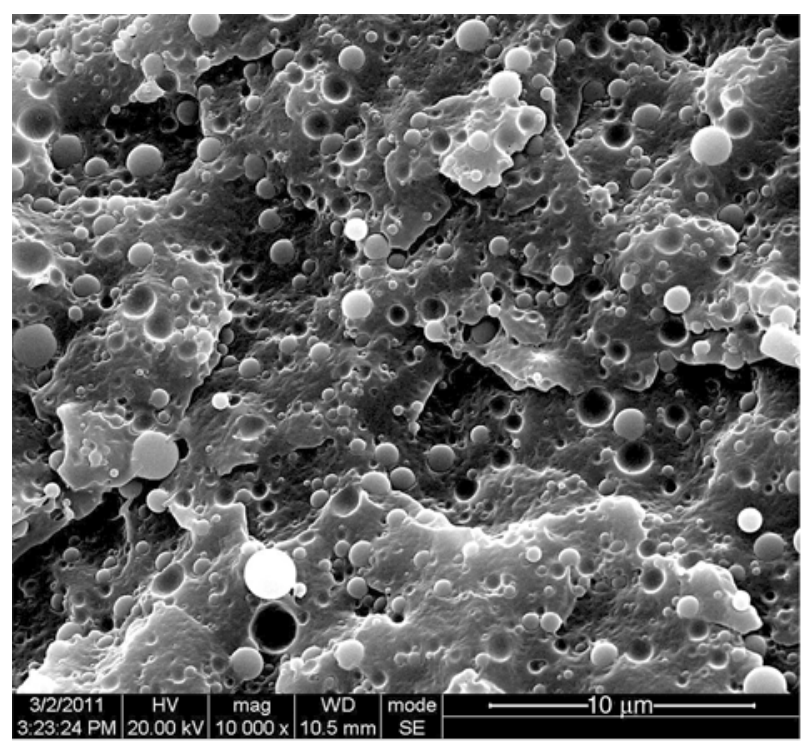

a)

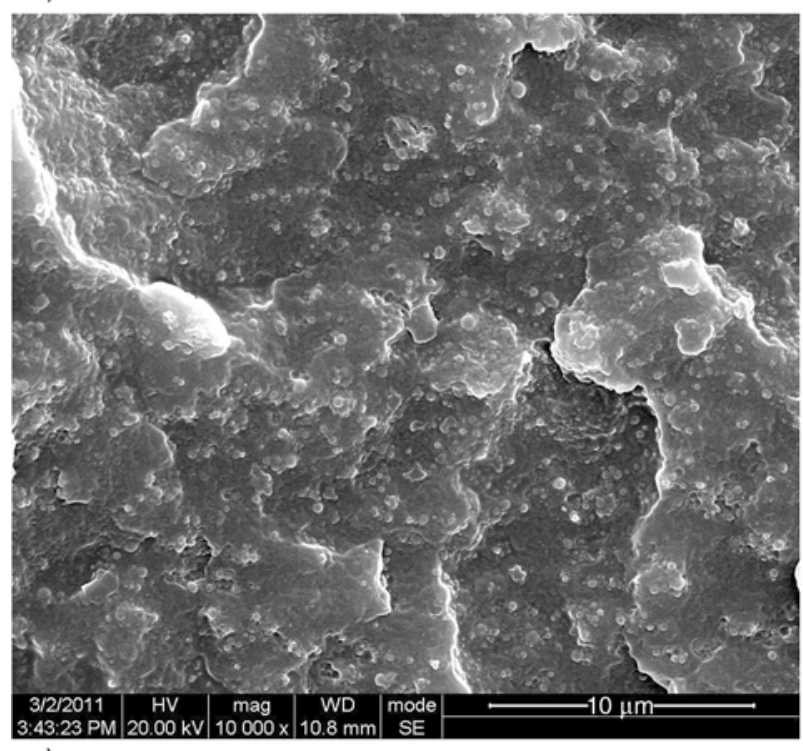

c)

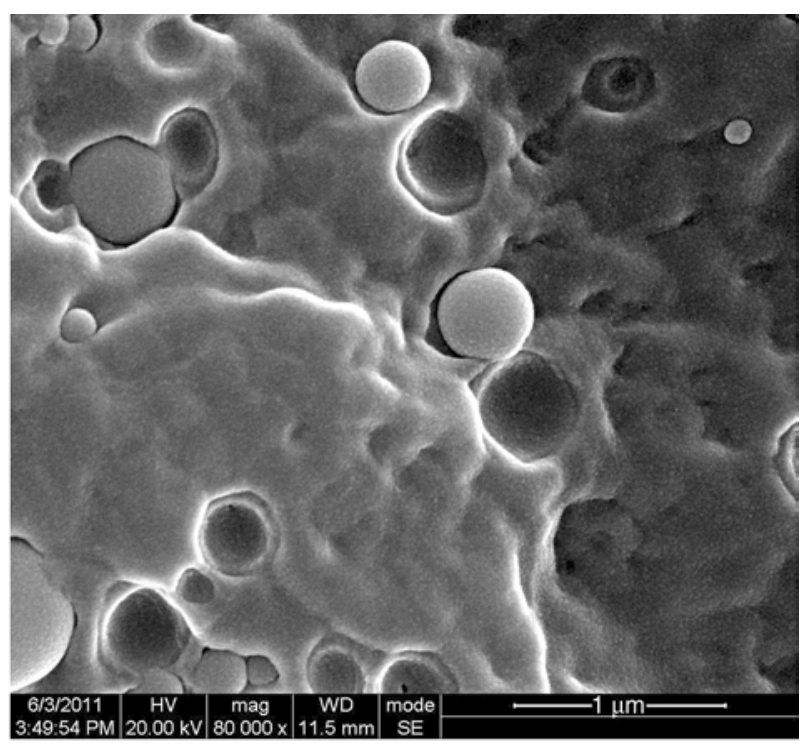

b)

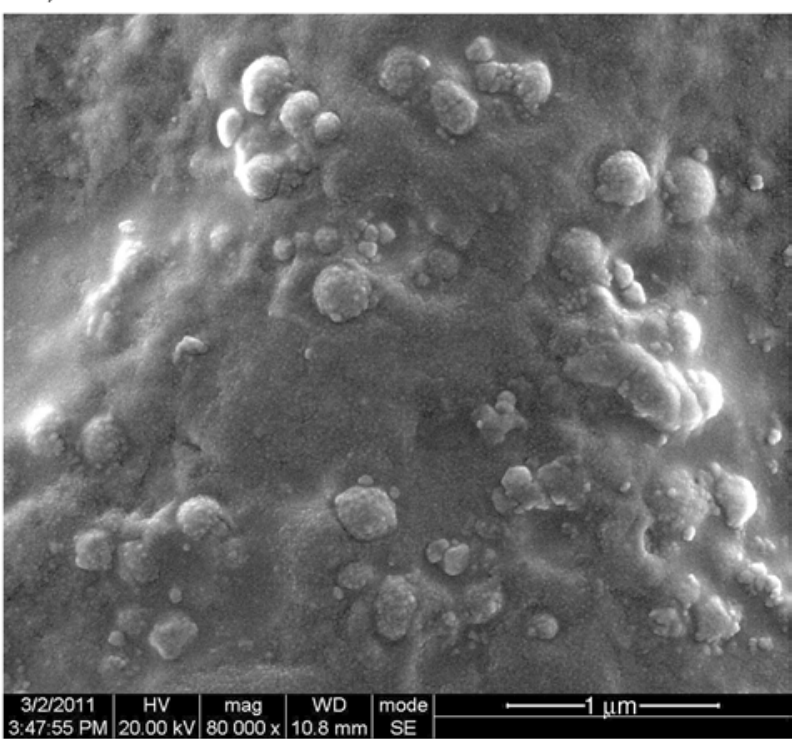

d)

Figure 3. SEM images of PVDF with $20 \% \mathrm{SiO}_{2}$ at low magnification (a) and high magnification (b); PVDF with $20 \%$ $\mathrm{SiO}_{2}-\mathrm{GO}$ at low magnification (c) and high magnification (d)

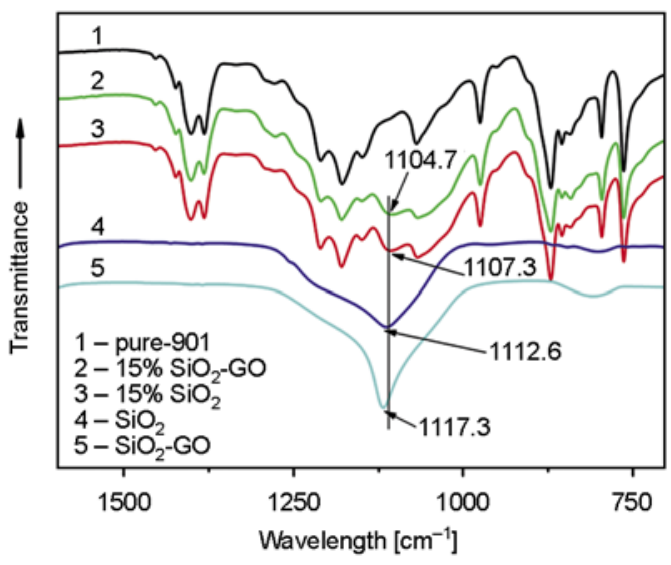

Figure 4. FTIR spectrums of PVDF nanocomposites and the fillers improve the interfacial behaviour and may have an effect on the mechanical properties of the PVDF/ $\mathrm{SiO}_{2}$-GO composites.

A decrease of the spherulite size, results in a stronger interaction between PVDF chains and nanoparticles [23]. The morphologies of crystals were shown in Figure 5. The slice of samples were relatively thick for the low melt index of PVDF, so the serious overlap of crystal in the picture was hard to avoid. Compared with pure PVDF, the spherulite size of PVDF in PVDF $/ 5 \% \mathrm{SiO}_{2}$ composite was larger, which indicated that the interaction between PVDF chains and $\mathrm{SiO}_{2}$ was weak. However, the spherulite size of PVDF in $\mathrm{PVDF} / 5 \% \mathrm{SiO}_{2}-\mathrm{GO}$ composite was obviously smaller than that of pure 


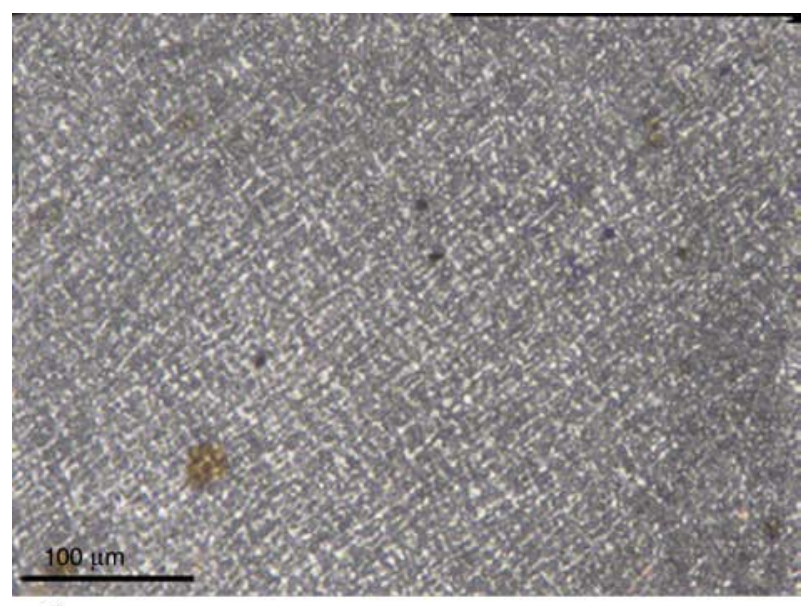

a)

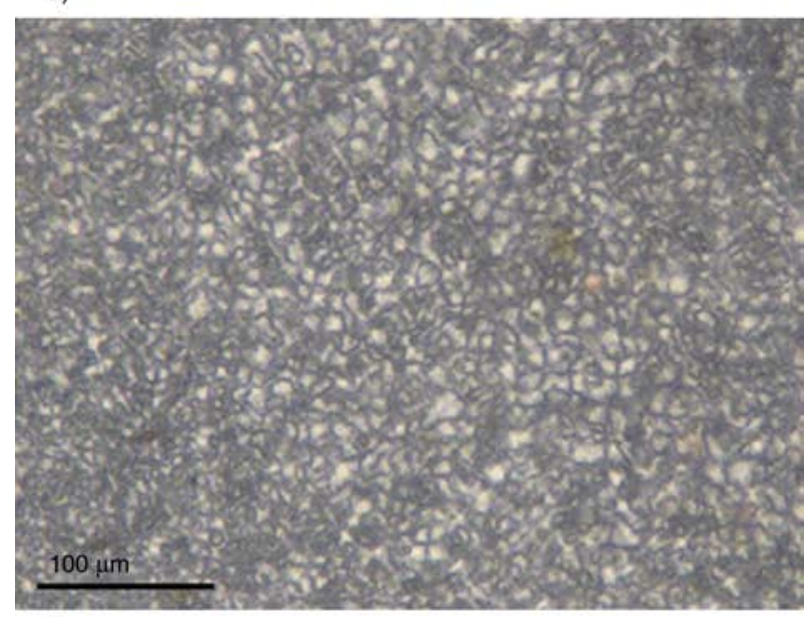

b)

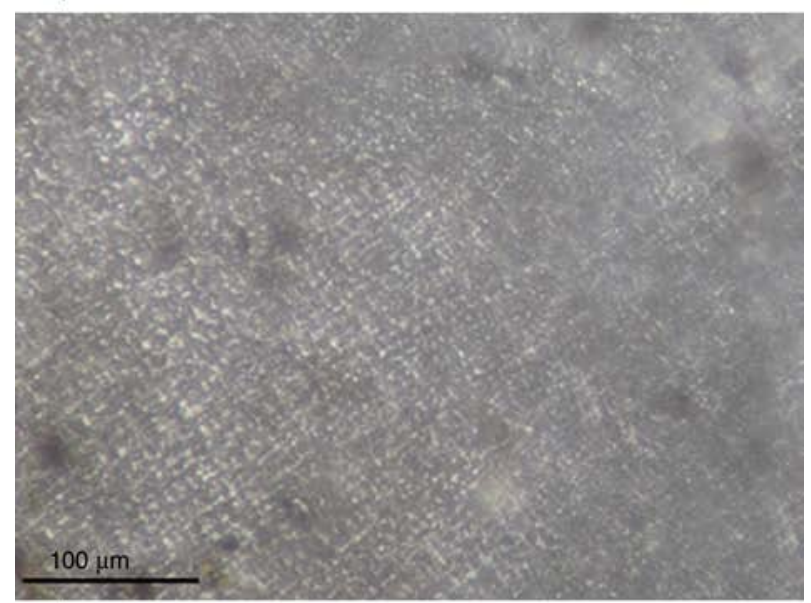

c)

Figure 5. Crystal morphologies of PVDF (a), PVDF with $5 \% \mathrm{SiO}_{2}(\mathrm{~b})$ and $5 \% \mathrm{SiO}_{2}-\mathrm{GO}(\mathrm{c})$ respectively observed by Polarized optical microscopy (POM)

PVDF, indicating a better interfacial interaction between PVDF chains and $\mathrm{SiO}_{2}$-GO particles.

In summary, it can be concluded that the interfacial interaction between PVDF and $\mathrm{SiO}_{2}-\mathrm{GO}$ has been largely improved compared with that between PVDF and silica. That is to say, after $\mathrm{SiO}_{2}$ particles were coated with graphene oxide, the interfacial interaction was greatly enhanced in $\mathrm{PVDF} / \mathrm{SiO}_{2}-\mathrm{GO}$ composites. It should be a potential method to improve the interfacial interaction between polymer and fillers.

\subsection{Mechanical properties of PVDF nanocomposites}

Since the interfacial interaction was enhanced in the $\mathrm{PVDF} / \mathrm{SiO}_{2}-\mathrm{GO}$ composites and graphene show high mechanical properties [2], the mechanical properties of $\mathrm{PVDF} / \mathrm{SiO}_{2}-\mathrm{GO}$ composites were supposed to be enhanced. Figure 6a showed tensile strengths of $\mathrm{PVDF} / \mathrm{SiO}_{2}$ and $\mathrm{PVDF} / \mathrm{SiO}_{2}-\mathrm{GO}$ composites. The tensile strength of $\mathrm{PVDF} / \mathrm{SiO}_{2}-\mathrm{GO}$ composites show a limited improvement compared with $\mathrm{PVDF} / \mathrm{SiO}_{2}$ composites with different filler contents. The tensile strength of pure PVDF was 45.6 $\mathrm{MPa}$, with increasing loading of $\mathrm{SiO}_{2}-\mathrm{GO}$, the tensile strength of $\mathrm{PVDF} / \mathrm{SiO}_{2}-\mathrm{GO}$ increased. The tensile strength of PVDF containing $20 \% \mathrm{SiO}_{2}-\mathrm{GO}$ was $51.2 \mathrm{MPa}$, which was about $12 \%$ more than that of pure PVDF. While the tensile strength of $\mathrm{PVDF} / \mathrm{SiO}_{2}$ decreased with the increasing loading of $\mathrm{SiO}_{2}$, it was subjected to severe decrease when the loading of $\mathrm{SiO}_{2}$ was $20 \%$, this may be due to the aggregation of $\mathrm{SiO}_{2}$ in PVDF matrix and poor interfacial interaction between PVDF and $\mathrm{SiO}_{2}$. The limited improvement of tensile strength could be ascribed to the good dispersion of $\mathrm{SiO}_{2}-\mathrm{GO}$ and interfacial enhancement between $\mathrm{SiO}_{2}$-GO nanoparticles and PVDF matrix. The basal planes of graphene oxide were most likely decorated with epoxide, hydroxyl, carbonyl and carboxyl groups. Thus, graphene oxide coated silica with high polarity dispersed well in polar PVDF. The good interaction between the functional groups of $\mathrm{SiO}_{2}-\mathrm{GO}$ and PVDF molecular chains would result in better interfacial interaction.

The tensile modulus is shown in Figure $6 \mathrm{~b}$. Tensile modulus of $\mathrm{PVDF} / \mathrm{SiO}_{2}-\mathrm{GO}$ and $\mathrm{PVDF} / \mathrm{SiO}_{2}$ both increased with the increasing amount of fillers, this increase could be attributed to the presence of silica, because it was a common phenomenon when a polymer was blended with rigid particles. We noticed that the modulus of $\mathrm{PVDF} / \mathrm{SiO}_{2}-\mathrm{GO}$ was slightly lower than that of $\mathrm{PVDF} / \mathrm{SiO}_{2}$. In order to confirm this experimental result, we did a dynamic mechanical analysis (DMA test). Figure 7 showed that stor- 

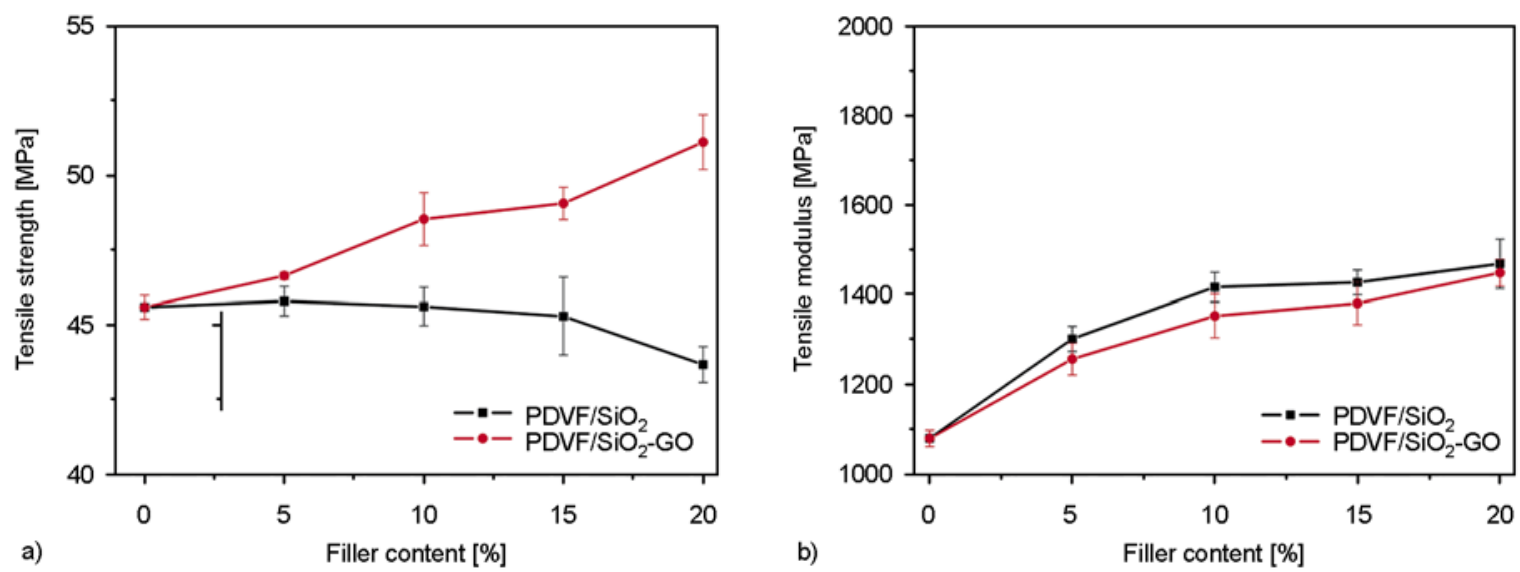

Figure 6. Tensile strength (a) and tensile modulus (b) of PVDF with $\mathrm{SiO}_{2}-\mathrm{GO}$ and $\mathrm{SiO}_{2}$ respectively

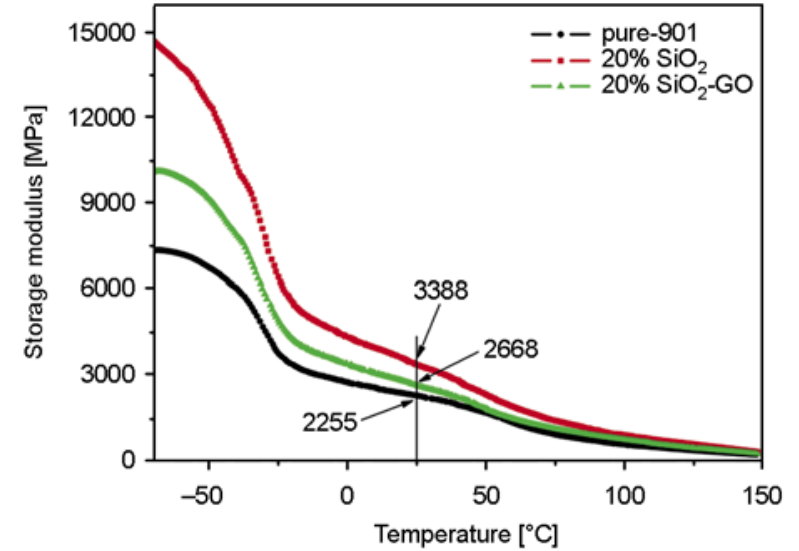

Figure 7. Storage modulus of PVDF with $\mathrm{SiO}_{2}-\mathrm{GO}$ and $\mathrm{SiO}_{2}$ respectively carried out by dynamic mechanical analysis (DMA) measurement

age modulus of $\mathrm{PVDF} / \mathrm{SiO}_{2}$ and $\mathrm{PVDF} / \mathrm{SiO}_{2}-\mathrm{GO}$ were obviously higher than that of pure PVDF. Even at $25^{\circ} \mathrm{C}$, the storage modulus of $\mathrm{PVDF} / \mathrm{SiO}_{2}$ (3388 MPa) was $50 \%$ higher than that of PVDF (2255 MPa), while the storage modulus of PVDF/ $\mathrm{SiO}_{2}$-GO $(2668 \mathrm{MPa})$ was only $18 \%$ higher than that of PVDF (2261 MPa). This result was similar to that of tensile tests, it confirmed that the rigid silica particle would result in an increase of modulus. However, the modulus increase of $\mathrm{PVDF} / \mathrm{SiO}_{2}-\mathrm{GO}$ was relatively lower than that of $\mathrm{PVDF} / \mathrm{SiO}_{2}$. Why did the tensile strength of $\mathrm{PVDF} / \mathrm{SiO}_{2}-\mathrm{GO}$ improved only by $12 \%$ at most and the modulus was relatively lower than that of $\mathrm{PVDF} / \mathrm{SiO}_{2}$ ? There were two possible explanations: 1) The APS layer between silica and graphene oxide may cause the rigidity of $\mathrm{SiO}_{2}$-GO to decrease, compared with the pure silica. Thus, the modulus of $\mathrm{PVDF} / \mathrm{SiO}_{2}-\mathrm{GO}$ was relatively lower than that of $\mathrm{PVDF} / \mathrm{SiO}_{2}$. 2) Recently, researchers proposed that the graphene oxide contains many oxidative debris after oxidation of natural graphite flakes [24]. Maybe the small molecules (oxidative debris) mixed with graphene oxide could act as plasticizer, thus do harm to the improvement of strength of $\mathrm{PVDF} / \mathrm{SiO}_{2}-\mathrm{GO}$ and result in the relatively lower modulus of $\mathrm{PVDF} / \mathrm{SiO}_{2}-\mathrm{GO}$.

\section{Conclusions}

Graphene oxide coated silica hybrids $\left(\mathrm{SiO}_{2}-\mathrm{GO}\right)$ were fabricated by electrostatic assembly, and $\mathrm{SiO}_{2}-$ GO hybrids based PVDF nanocomposites were made successfully. SEM pictures showed that there were many $\mathrm{SiO}_{2}$ particles pulled out of the fracture surface of PVDF/ $/ \mathrm{SiO}_{2}$, the interface between PVDF matrix and $\mathrm{SiO}_{2}$ was clear. While for $\mathrm{PVDF} / \mathrm{SiO}_{2}-$ $\mathrm{GO}$, there were few $\mathrm{SiO}_{2}$-GO particles pulled out of the fracture surface, $\mathrm{SiO}_{2}$-GO particles were mostly embedded in the PVDF matrix, the interface between PVDF matrix and $\mathrm{SiO}_{2}-\mathrm{GO}$ was unclear. This indicated that the interfacial interaction between PVDF matrix and $\mathrm{SiO}_{2}-\mathrm{GO}$ was greatly enhanced. Using infrared spectra investigation, the vibration peak of silica in $\mathrm{PVDF} / \mathrm{SiO}_{2}-\mathrm{GO}$ showed an obvious 'red shift' compared with $\mathrm{SiO}_{2}-\mathrm{GO}$, indicating the strong hydrogen bonds forming between PVDF matrix and $\mathrm{SiO}_{2}-\mathrm{GO}$, which will be beneficial for the interfacial enhancement. Compared with neat PVDF, crystallite size of $\mathrm{PVDF} / \mathrm{SiO}_{2}-\mathrm{GO}$ decreased, while those of $\mathrm{PVDF} / \mathrm{SiO}_{2}$ increased. This indicated that, compared with $\mathrm{PVDF} / \mathrm{SiO}_{2}, \mathrm{PVDF} / \mathrm{SiO}_{2}-\mathrm{GO}$ had a better interfacial interaction. Since the interface was greatly enhanced in $\mathrm{PVDF} / \mathrm{SiO}_{2}-\mathrm{GO}$ composites, the corresponding mechanical properties were exam- 
ined. The tensile strength of PVDF/ $\mathrm{SiO}_{2}-\mathrm{GO}$ showed limited improvement, and modulus was relatively lower, compared with $\mathrm{PVDF} / \mathrm{SiO}_{2}$.

\section{Acknowledgements}

We express our sincere thanks to the Program for the NewCentury Excellent Talents of Ministry of Education of China (NCET-10-0580) for financial support.

\section{References}

[1] Novoselov K. S., Geim A. K., Morozov S. V., Jiang D., Zhang Y., Dubonos S. V., Grigorieva I. V., Firsov A. A.: Electric field effect in atomically thin carbon films. Science, 306, 666-669 (2004). DOI: $10.1126 /$ science. 1102896

[2] Lee C., Wei X., Kysar J. W., Hone J.: Measurement of the elastic properties and intrinsic strength of monolayer graphene. Science, 321, 385-388 (2008). DOI: $10.1126 /$ science. 1157996

[3] Rao C., Sood A., Subrahmanyam K., Govindaraj A.: Graphene: The new two-dimensional nanomaterial. Angewandte Chemie International Edition, 48, 77527777 (2009).

DOI: $10.1002 /$ anie. 200901678

[4] Balandin A. A., Ghosh S., Bao W., Calizo I., Teweldebrhan D., Miao F., Lau C. N.: Superior thermal conductivity of single-layer graphene. Nano Letters, 8 , 902-907 (2008).

DOI: $10.1021 / \mathrm{n} 10731872$

[5] Schniepp H. C., Li J-L., McAllister M. J., Sai H., Herrera-Alonso M., Adamson D. H., Prud'homme R. K., Car R., Saville D. A., Aksay I. A.: Functionalized single graphene sheets derived from splitting graphite oxide. The Journal of Physical Chemistry B, 110, 8535-8539 (2006).

DOI: $10.1021 / \mathrm{jp} 060936 \mathrm{f}$

[6] Cai W., Piner R. D., Stadermann F. J., Park S., Shaibat M. A., Ishii Y., Yang D., Velamakanni A., An S. J., Stoller M., An J., Chen D., Ruoff R. S.: Synthesis and solid-state NMR structural characterization of ${ }^{13} \mathrm{C}$ labeled graphite oxide. Science, 321, 1815-1817 (2008).

DOI: $10.1126 /$ science.1162369

[7] Kim F., Cote L. J., Huang J.: Graphene oxide: Surface activity and two-dimensional assembly. Advanced Materials, 22, 1954-1958 (2010).

DOI: $10.1002 / \mathrm{adma} .200903932$

[8] Kim J., Cote L. J., Kim F., Yuan W., Shull K. R., Huang J.: Graphene oxide sheets at interfaces. Journal of the American Chemical Society, 132, 8180-8186 (2010).

DOI: $\underline{10.1021 / j a 102777 p}$
[9] Fang M., Wang K., Lu H., Yang Y., Nutt S.: Covalent polymer functionalization of graphene nanosheets and mechanical properties of composites. Journal of Materials Chemistry, 19, 7098-7105 (2009).

DOI: 10.1039/B908220D

[10] Kou L., Gao C.: Making silica nanoparticle-covered graphene oxide nanohybrids as general building blocks for large-area superhydrophilic coatings. Nanoscale, $\mathbf{3}$, 519-528 (2011). DOI: $10.1039 / \mathrm{c} 0 \mathrm{nr} 00609 \mathrm{~b}$

[11] Li N., Liu G., Zhen C., Li F., Zhang L., Cheng H-M.: Battery performance and photocatalytic activity of mesoporous anatase $\mathrm{TiO}_{2}$ nanospheres/graphene composites by template-free self-assembly. Advanced Functional Materials, 21, 1717-1722 (2011).

DOI: 10.1002/adfm.201002295

[12] Yang S., Feng X., Ivanovici S., Müllen K.: Fabrication of graphene-encapsulated oxide nanoparticles: Towards high-performance anode materials for lithium storage. Angewandte Chemie International Edition, 49, 84088411 (2010).

DOI: 10.1002 /anie.201003485

[13] Lovinger A.: Ferroelectric polymers. Science, 220, 1115-1121 (1983).

DOI: $10.1126 /$ science.220.4602.1115

[14] Wang M., Shi J-H., Pramoda K. P., Goh S. H.: Microstructure, crystallization and dynamic mechanical behaviour of poly(vinylidene fluoride) composites containing poly(methyl methacrylate)-grafted multiwalled carbon nanotubes. Nanotechnology, 18, 235701/1-235701/7 (2007).

DOI: 10.1088/0957-4484/18/23/235701

[15] Yee W. A., Kotaki M., Liu Y., Lu X.: Morphology, polymorphism behavior and molecular orientation of electrospun poly(vinylidene fluoride) fibers. Polymer, 48, 512-521 (2007). DOI: $10.1016 /$ j.polymer.2006.11.036

[16] Casciola M., Capitani D., Donnadio A., Diosono V., Piaggio P., Pica M.: Polyvinylidene fluoride/zirconium phosphate sulfophenylphosphonate nanocomposite films: Microstructure and mechanical properties. Journal of Materials Chemistry, 18, 4291-4296 (2008). DOI: 10.1039/B807948J

[17] Chen D., Wang M., Zhang W-D., Liu T.: Preparation and characterization of poly(vinylidene fluoride) nanocomposites containing multiwalled carbon nanotubes. Journal of Applied Polymer Science, 113, 644650 (2009).

DOI: 10.1002/app.29311

[18] Shah D., Maiti P., Gunn E., Schmidt D. F., Jiang D. D., Batt C. A., Giannelis E. P.: Dramatic enhancements in toughness of polyvinylidene fluoride nanocomposites via nanoclay-directed crystal structure and morphology. Advanced Materials, 16, 1173-1177 (2004). DOI: $10.1002 / \mathrm{adma} .200306355$ 
[19] Tran M. Q., Shaffer M. S. P., Bismarck A.: Manufacturing carbon nanotube/PVDF nanocomposite powders. Macromolecular Materials and Engineering, 293, 188-193 (2008).

DOI: $10.1002 /$ mame.200700340

[20] Kim J-W., Cho W-J., Ha C-S.: Morphology, crystalline structure, and properties of poly(vinylidene fluoride)/ silica hybrid composites. Journal of Polymer Science Part B: Polymer Physics, 40, 19-30 (2002).

DOI: $10.1002 /$ polb.10071

[21] Hummers W. S., Offeman R. E.: Preparation of graphitic oxide. Journal of the American Chemical Society, 80, 1339 (1958).

DOI: $10.1021 / \mathrm{ja} 01539 \mathrm{a} 017$
[22] Hong J., Char K., Kim B-S.: Hollow capsules of reduced graphene oxide nanosheets assembled on a sacrificial colloidal particle. The Journal of Physical Chemistry Letters, 1, 3442-3445 (2010). DOI: $10.1021 / \mathrm{jz} 101441 \mathrm{a}$

[23] Ma W., Wang X., Zhang J.: Effect of MMT, $\mathrm{SiO}_{2}$, $\mathrm{CaCO}_{3}$, and PTFE nanoparticles on the morphology and crystallization of poly(vinylidene fluoride). Journal of Polymer Science Part B: Polymer Physics, 48, 2154-2164 (2010).

DOI: $10.1002 /$ polb.22097

[24] Rourke J. P., Pandey P. A., Moore J. J., Bates M., Kinloch I. A., Young R. J., Wilson N. R.: The real graphene oxide revealed: Stripping the oxidative debris from the graphene-like sheets. Angewandte Chemie, 123, 32313235 (2011).

DOI: $10.1002 /$ anie. 201007520 